\title{
IoT and Public Weather Data Based Monitoring \& Control Software Development for Variable Color Temperature LED Street Lights
}

\author{
G.B. Satrya ${ }^{\# *}$, H.T. Reda ${ }^{\#}$, J.W. Kim ${ }^{\#}$, P.T. Daely", S.Y. Shin" ${ }^{\#}$ S. Chae \\ ${ }^{\#}$ Department of IT Convergence, Kumoh National Institute of Technology, Gyeongbuk, 39177, Republic of Korea \\ "Telkom Applied Science School, Telkom University, Bandung, 40257, Republic of Indonesia \\ E-mail: \{gandevabs, haft2, rerua, 20156130, khaira.latif,wdragon, schae\}@kumoh.ac.kr
}

\begin{abstract}
Light Emitting Diode (LED) is quickly becoming the latest technology in the application of lighting on streets. By combining the concept of Internet of Things (IoT) and LED streetlight, this paper aims to apply the concept of microservices architecture so that the development process can be agile and scalable on-demand. This paper also describes the integration between the hardware of LED and Application Program Interface (API) SKPlanet, Airkorea and OpenStreetMap then displays it to the user via a web browser on PC or on mobile via HTTP access. Furthermore, we implemented the monitoring of a smart sensor interface with the Internet can be seen from the administrator or smartphone.
\end{abstract}

Keywords - internet of things (IoT); wireless sensor networks (WSN); smart LED; streetlight system; LED; software development

\section{INTRODUCTION}

Traditional High-Pressure Sodium (HPS) based street lamps have been replaced by Light Emitting Diodes (LED) lamps for the latter can offer more substantial energy savings than the former. Nowadays, many developed nations are committed to the transformation of their cities into smart cities partly by introducing energy-efficient streetlight system. Furthermore, making the street LED lamps smart can further bring tremendous energy savings. Especially, the use of weather data aware correlated colour temperature (CCT) based smart LEDs in streetlights system will be a tremendous achievement towards building an energy efficient ecosystem and building a platform of smart city services such as the smart streetlight system which is our subject in this article.

According to Lopez Research [1] Internet of Things (IoT) defines a system where things in the physical world and sensors within or in close proximity to these things are connected to the Internet by means of either wireless or wired Internet connectivity. The main target of IoT is to extend the benefits of Internet connectivity of several other interconnected devices [2] [3]. It is highly expected that IoT will unify plenty of applications such as wearables, smart home, smart city, and smart streetlight systems into the Internet. Although several communication technologies are known to many IoT applications wireless sensor networks (WSN) remains the well-known candidate towards wireless based IoT. WSN is the most important element of IoT thereby providing information regarding the physical world. In this paper, we study several wireless sensors installed on the LED streetlight and their connection to the internet. We perform periodical monitoring activities of the installed wireless sensor based LED streetlights to receive the latest information as well as to control their status remotely.

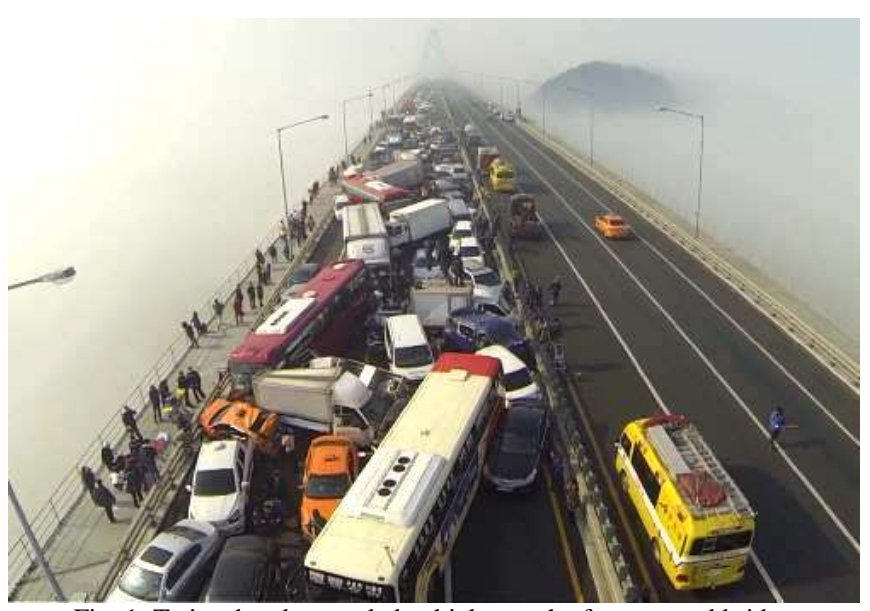

Fig. 1 Twisted and crumpled vehicles on the fog-covered bridge

The main purpose of streetlights is to illuminate the roads mainly during night time and at severe weather conditions such as very cloudy or snowy day time. Previously, in a streetlight system, white LEDs have been given due 
consideration as a compliment for HPS. However, recently, the study of CCT [4] of a LED for different luminance requirements of different roads. The colour temperature - or the CCT - is descriptive of the colour of emitted light [5]. Colour temperatures over $5000 \mathrm{~K}$ are called cool colours (blueish white), while lower colour temperatures of $2700 \mathrm{~K}$ to $3000 \mathrm{~K}$ are called warm colours (yellowish white through red).

As a requirement for our project work of this paper, we consider variable colour temperature to consider the effect of harsh weather data such as fog, rain, snow. In particular, a $5.000 \mathrm{~K}$ and $3.000 \mathrm{~K}$ based colour temperature of lamp improves the road perceive ability. Hence, this project is implemented web-based to collect climate data such as fog, rain, snow, and etc. according to our project requirements. Furthermore, it is required that an integrated streetlight system should prevent traffic accidents. Therefore, we develop a software-based management system to meet the aforementioned requirement. For this reason, the LED is integrated with the software management system for operational and management of road lighting. This ensures that the system can control and communicate with hardware and software.

Our motivation to develop this system is in order to detect a driver's blind spot when climate alarm issue (fog, rain, snow, etc.) will cause traffic accidents due to low visibility. Typically, such traffic accident has happened on a foggy day, like the 106 vehicles chain collision accident on Yeongjong Bridge, South Korea in February 2015 as shown in Fig. 1 [6]. A study in [4] shows improvement of visibility on the foggy street around $4 \%$ when the CCT of streets is changed from $5000 \mathrm{~K}$ to $3000 \mathrm{~K}$ and through which the probability of street accidents, similar to the Youngjong Grand bridge chain-reaction collision accident, can be lowered somehow. So, by collecting the climate data from the web and/or public weather data, not only can we change the colour temperature of LED streetlight dynamically, but also can we improve the visibility of car drivers thereby improving the prevention of the aforementioned traffic accidents.

Besides, in this paper, we use java servlet based application servers. Our software development process involves using agile concepts in programming and connects with microservices architecture [7], [8]. The use of agile concept here is to ease the development of the software in the long term if something needs to be added or removed from the plan. This is contrary to the concept of the traditional approach of a waterfall in which the process should be taken sequentially and takes a long time in its reconstruction. Meanwhile, the microservice architecture is used to improve the available software system. This is very useful for the development of IoT to enable all the connections that exist inside a complex architecture. Microservices used in this paper is SKPlanet, AirKorea, OpenStreet Map.

The rest of the paper is organized as follows. In section II we discuss related works and include our contribution of this paper showing the difference with existing works. Section II also provides a thorough discussion of methodology, system design, and architecture used in the implementation. In section III, we talk about the process of analysis, results, and discussion. Finally, we conclude our article in section IV.

\section{MATERIALS AND METHODS}

In this section, we comprehensively discuss the literature review, research methodology, and network design aspects of our article.

\section{A. Literature Review}

The design of smart and intelligent road lamps offers energy efficiency, extends the lifetime of the lighting equipment, and easy maintenance [9]. In [10] implementation of low-cost sensors and embedded controller based dynamic street lights is proposed to reduce the energy consumption. Traffic flow based smart LED streetlight system is proposed [11] to deliver substantial energy savings in response to adaptive traffic on the street.

WSNs are more advantageous than any other communication networks for remote LED streetlight monitoring [12]. The design of remote streetlight monitoring using WSNs is considered in [13]. In this work, the central control unit is used to monitor streetlight system, display topology and location maps, and send control messages to remote units, thereby gathering information of each streetlight. The sensor node in this work is used to turn the streetlight on and off, and also for dimming functionality according to the received messages from the remote unit. Mendalka et al. [14] proposed to apply WSN to intelligent street lighting system concept based. The proposed system is to provide light in streets and public places (e.g. parking lots, gas stations) with the required quality. All lamp events delivered to the lamp commissioner will provide valuable information about system's activity, performance, and potential malfunctions.

Leccese and Leonowicz [15] proposed an intelligent wireless street lighting system. It consists of measuring stations (lamppost consists of many sensors), presence detector (identifying the passage of a vehicle or pedestrian), light sensor (measure the external light intensity to assure a minimum level of illumination of the road), Supervision module (which improves sensor fault management and system maintenance), Management centre (the hub of the system, since it permits the visualization and control of the complete lighting system). Moreover, Lian and Li [16] proposed control network. Their research focuses on reducing the power consumption, decrease the management cost and monitor the status information of each street lighting unit. The methods of LED dimming mainly contain three as follows: first is dimming technology for regulating the forward direction current, second is dimming with pulse width modulation, and third is LED dimming using thyristor.

Although different WSNs have been used for remote LED streetlight control applications, ZigBee is one of the potential candidates. ZigBee network has been used for LED streetlight control system, including different frameworks and network node deploying ability. Authors in [17] and [18] proposed smart streetlight system which is based on turning the streetlights on or off whenever motion is detected or not under specific area. They considered power adjustable LED lamps, motion and brightness sensors as well as ZigBee based wireless communication modules. In [19] and [20], the authors proposed LED streetlight remote control system which is implemented through a network of sensors to gather information relevant to the management, and transmit the 
information via ZigBee network. Other works in [21] and [22] show that the use of ZigBee network for communication can mitigate energy consumption of streetlight.

Other works such as [23] propose a platform design with $\mathrm{CCT}$ control based on weather condition using ZigBee. The design is similar to our proposed design, but there is no explanation about the remote interface for an administrator to control the streetlight. In [24] and [25], the authors show how to control the CCT of streetlight by reading the weather condition from weather application programming interface, but the streetlights do not give any local weather data to the server. More thorough work is shown in [26], where the request and reply communication is implemented. The use of website and smartphone application is used to display the status of the streetlights.

IoT is the term we now use for Internet usage where the Internet now gives effect to education, communication, business, science, government, and with things that our lives depend on [2], [3]. In other words, the connection of multiple hardware and software that uses the Internet connection and can be used by everyone (open access) or some group (closed service). In 2015, there are about 25 billion devices connected to the Internet and predicted that 2020 would rise to double to 50 billion devices will be connected.

Although there is no standard definition for the microservices format, there are certain characteristics that aid in the identification of style [7], [8]. Basically, microservices architecture is a method of software application development as a suite of independent deployability, small, modular services where each service runs unique processes and communicate via a clear mechanism, mild in serving business goals. As mentioned above, we use Application Program Interface (API) in three different microservices:

- For microservice SKPlanet, we will adopt the weather information, latitude, and longitude.

- For microservice AirKorea, we will adopt PM10 dust concentration.

And for OpenStreet Map we are adopting open source map to map allocation point where the sensor is located.

\section{B. Research Methodology}

In software engineering, there are two basic methodologies: Waterfall (aka "traditional approach") and Agile.

1) Waterfall: Waterfall method, despites being very ancient and traditional method, is still better approach to use [27], [28]. If the software production requires a clear and non-sophisticated requirement, waterfall approach is still the best option. Some of the shortcomings of the waterfall method are: First, the severe problem could result if not handled properly or known from the beginning of software development. For this reason, proper project management is required to minimize the error. Second, the system requirements must be described clearly. Moreover, process details must be absolutely clear and should not be changed periodically. Third, it is difficult to adapt to the specifications when certain changes are required at any stage of the development. Therefore, changes in the midst of the work will confuse the team work. The typical waterfall development cycle is depicted in Fig. 2.

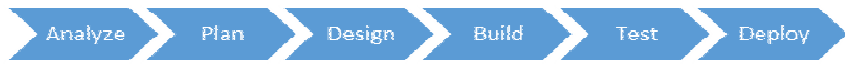

Fig. 2 The waterfall development cycle

2) Agile: This method is iterative and underlines a rapid application delivery in complete functional constituents. In this case, the application developed using this methodology of the development cycle is agile. The word, 'Agile' has the origins from the Latin word which has the meaning 'flexibility'. This methodology was chosen to develop applications and to quickly deliver the necessary user to continuous improvement (as shown in Fig. 3). By using such method, applications can be delivered in stages without waiting for all the requirements to complete [27], [28]. So this approach can also improve client satisfaction more than the traditional method. Customers can conduct a review of the software created earlier and made faster system development. Therefore, it can reduce the risk of failure of the software implementation of non-technical terms. Different agile methods and the challenges towards software development process is highlighted in [29]. Moreover, authors in [30] proposed an iterative generalized estimated method and introduced case study based algorithm for the agile methods.

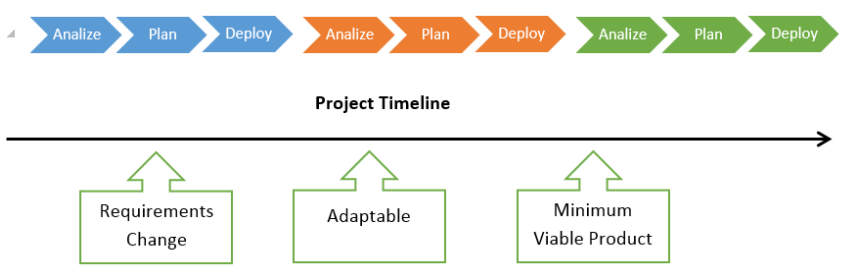

Fig. 3 The agile development cycle

So we choose Agile for the design and software development cycle.

\section{Network Topology}

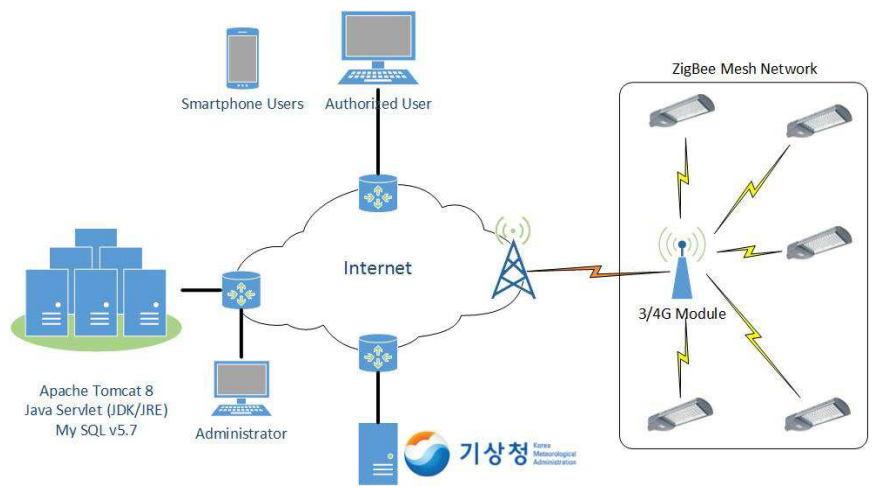

Fig. 4. Design network topology for system monitoring and configuration

Fig. 4 above is a topology in the implementation part that we apply. We used java application server based on Tomcat servlet 8, and for the database, we used MySQL 5.7. In the hardware side, we used ZigBee-based sensors connected to the existing LED streetlight streets. Users can see directly from the administrator or smartphone. We provide a 
username/password for accessing the system. For this purpose, we suggest the IoT and public weather data based monitoring and control software development for variable colour temperature LED street lights.

The suggested system consists of four major components,

1) Colour Temperature Varying LED Streetlight: As mentioned in the introduction section the monitoring system can provide an automatic command to the hardware when the weather changes or disasters. Based on data from humidity, PM10, and weather warning, we will change the colour temperature of the LED. For example, if there is an update of Korean weather API such as heavy snow, then the system will automatically give commands to the hardware in accordance with the GPS coordinates of the data to change the LED with the initial condition $5 \mathrm{~K}$ became $3 \mathrm{~K}$.

2) Public Weather Data API: It extracts climate data from the web and/or public weather data networks and delivers to remote control/monitoring centre for LED streetlights. By the initial conditions, the admin must register all hardware LEDs located on the street in accordance with their respective GPS coordinates. This system will match the GPS data and coordinate data Korean Weather API to provide updates of temperature and humidity. The system will provide updates every minute of Korean open API SKPlanet.

3) Remote Control/Monitoring System Server: The system can provide information automatically as well as it performs controlling. We can do monitoring 24 hours/ 7 days from a computer or smartphone. This system can also make changes if necessary. And these systems can also provide commands directly to the coordinates of the point where the LED's are like information about voltage, current, temperature, and humidity.

4) Communication between LED streetlights and Server: For communication, we use the principles of TCP / IP socket which is based on the client and server. Each LED streetlight sensors should know the IP address of the server. In our system, the communication procedure is divided into 2 parts. First, LED Street lights to the server where LEDs can provide parameters such as dimming, voltage, current, temperature, and humidity. Here, the server (web-based) will display the content online in the form of web-based. Second, Server to LED street lights where the server can request the data related to the previously mentioned parameters and also can turn off and turn on LED street lights directly.

\section{System Architecture}

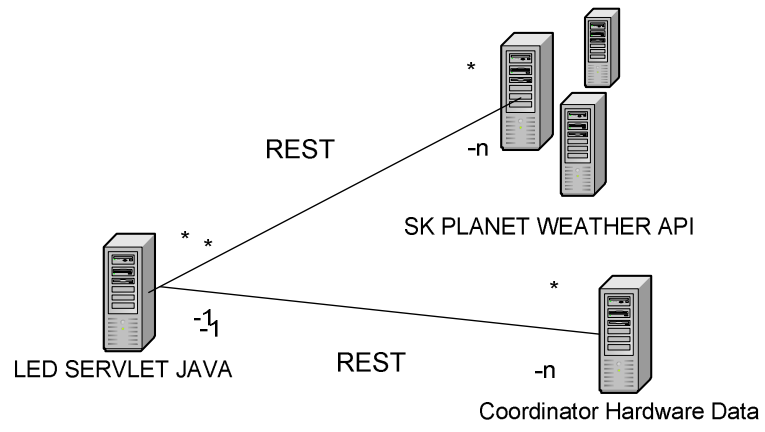

Fig. 5 System software architecture

In this subsection, we introduce and give a brief overview of the software design. The system architecture is a way to give the overall view of a system and to place it into context with external systems (as shown in Fig. 5). In order to orient users well and for easy readability we use three main interfaces as follows.

1) User Interfaces: The user interface for the system will allow the user to show Sensor/lamp geolocation data, and modify lamp. The user should be presented with all main functions on the first user interface page to allow for the user to select the function to use without the need to navigate inward to find it. It will be accessible through a web interface to allow for centralized hosting and use by various operating systems.

2) Software Interfaces: The software will need to interface with a case management system to pull data from it and push data updates to it. The connection will be a standard database connection using JDBC or ODBC.

3) Communication Interfaces: The software will need to interface with a case management system to pull data from it and push data updates to it. The connection will be a MySQL Database connection using JDBC or ODBC.

\section{E. Design GUI}

\begin{tabular}{|c|c|c|c|c|c|c|c|c|c|c|}
\hline Group_ID & $\begin{array}{l}\text { LED_ } \\
\text { DD }\end{array}$ & Dimming & Volatage & Current & Temperature & Humidity & Config & Long & Lat & $\begin{array}{l}\text { Weather } \\
\text { Info }\end{array}$ \\
\hline \multirow[t]{3}{*}{ Daejon } & & & & & Korean API & $\begin{array}{l}\text { Korean } \\
\text { API }\end{array}$ & & & & $\begin{array}{l}\text { Korean } \\
\text { API }\end{array}$ \\
\hline & 001 & Sensor & Sensor & Sensor & Sensor & Sensor & & & & \\
\hline & 002 & Sensor & Sensor & Sensor & Sensor & Sensor & & & & \\
\hline \multirow[t]{3}{*}{ Suwon } & & & & & Korean API & $\begin{array}{l}\text { Korcean } \\
\text { API }\end{array}$ & & & & $\begin{array}{l}\text { Korean } \\
\text { API }\end{array}$ \\
\hline & 001 & Sensor & Sensor & Sensor & Sensor & Sensor & & & & \\
\hline & 002 & Sensor & Sensor & Sensor & Sensor & Sensor & & & & \\
\hline \multirow[t]{3}{*}{ Gumi } & & & & & Korean API & $\begin{array}{l}\text { Korean } \\
\text { API }\end{array}$ & & & & $\begin{array}{l}\text { Korean } \\
\text { API }\end{array}$ \\
\hline & 001 & Sensor & Sensor & Sensor & Sensor & Sensor & & & & \\
\hline & 002 & Sensor & Sensor & Sensor & Sensor & Sensor & & & & \\
\hline
\end{tabular}

Fig. 6 Design GUI for LED street light

Fig. 6 shows the main page of the system where monitoring and configuration have been made. The system consists of 11 columns that will collaborate with three microservices which will be connected with some hardware LED Street light. As we can see from Fig. 6 each column title on the first line has a different function. For the second row, we make a group based on the largest cities in this area. We collect temperature, humidity, and the weather information (dust, rain, fog, snow, etc.) from Korean Weather API. On the third line, we get information of the LED street light sensor such as dimming, voltage, current, temperature, and humidity from the sensors already installed on the street.

\section{RESULTS AND DISCUSSION}

Here, we discuss the software system implementation and results achieved therein.

\section{A. Software System Overview}

1) Login Form: This feature provides user privilege through username/password. The purpose of this page is for security issue to give the user privilege limitation. In this system, two kinds of accesses are provided. One is an administrator who has full access. And the second is a public user with limited access or just can only access for viewing the system. Fig. 7 shows the login form used in our software management system. 


\section{Login with Username and Password}

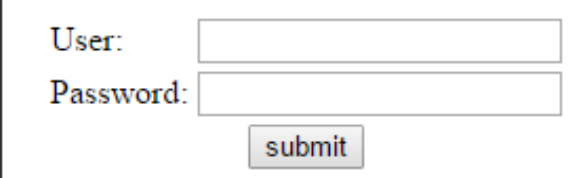

Fig. 7 Screenshot for login page

2) Main Form (Lamp Connection List): This page is the main page after login process in the system. In this page, the registered streetlights are listed (as shown in Fig. 8). As we mention in GUI design in the previous section, this system is already integrated with three microservices. Besides, this system is available to deliver the network connectivity status. For example, if there is a problem as shown in the third column by the red color which implies a problem in the hardware and the network. Similarly, the green color implies the hardware and network run well. On the right side, there is a Google street view (open source map) which shows the LED coordinate in the streetlight.

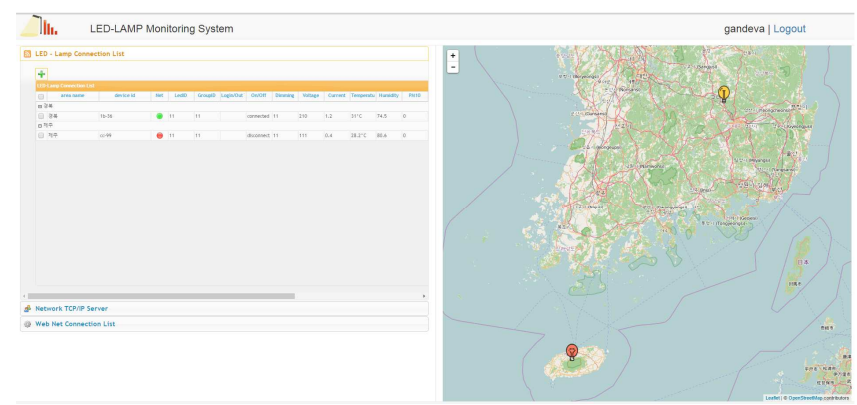

Fig. 8 Screenshot for the main page

3) Network Condition Form: This page provides data such as MAC address, group ID, led ID, IP address and status for checking in each LED Street light. "Connected" status refers to the system running well in the present, and "Disconnect" status means that the system has a problem (as shown in Fig. 9).

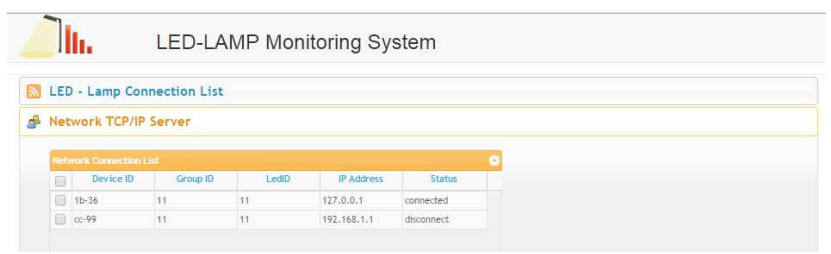

Fig. 9 Screenshot for network condition page

4) Creating/Editing Lamp Form: In this page, there are two parts. The first part is for inputting the new LED street light information which consists of LED ID, longitude and latitude. The second part is for updating process if needed such as coordinate, position and LED ID.

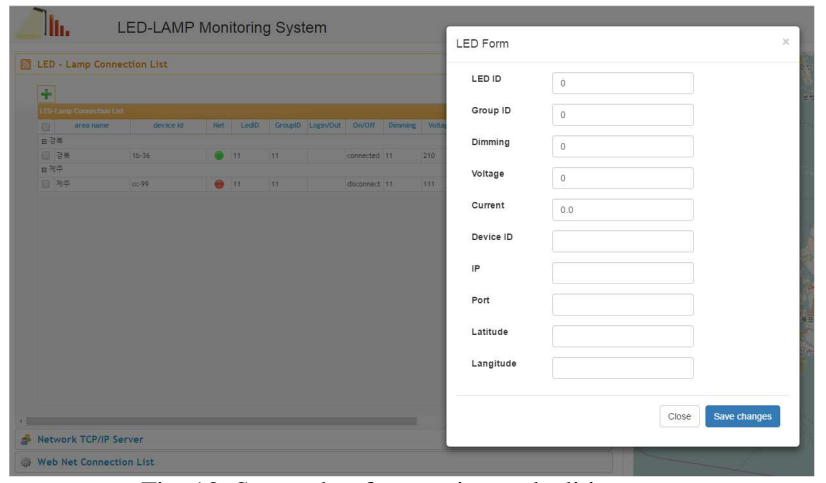

Fig. 10 Screenshot for creating and editing page

5) Request Form (Server to Hardware Communication): This system provides the information in monitoring system and configuration. This system is also available for sending a request to other sensors in LED Street light as can be shown in Fig. 11, of the first page of the request process. The most important thing in this process is knowing the number of LEDs which will be requested, then directly click the submit button.

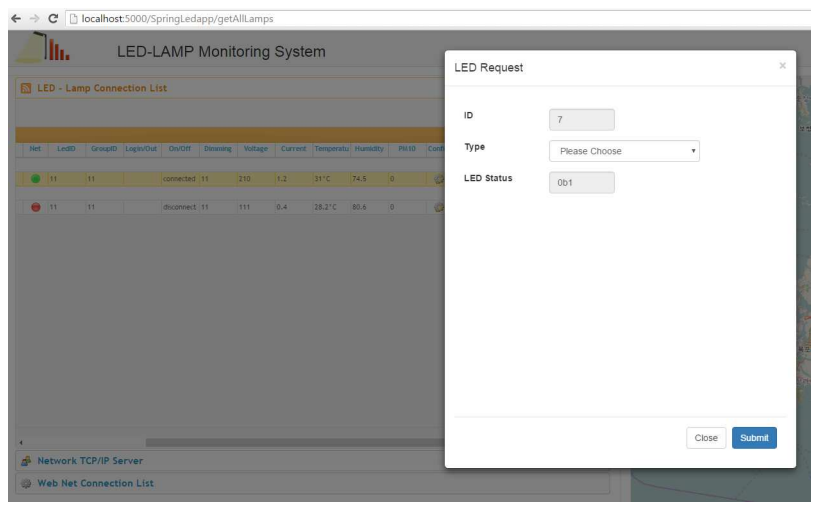

Fig. 11 Screenshot for request to the hardware page

6) Type of Request Option (Server to Hardware): In this page, we limit the input mode using the drop down method in order to reduce any human error. We give six choices depending on the project requirement as shown in Fig. 12. There are namely, LED Control ON, LED Control OFF, Request Temperature \& Humidity, Request Voltage \& Current, Request GPS, and Request Dust Concentration.

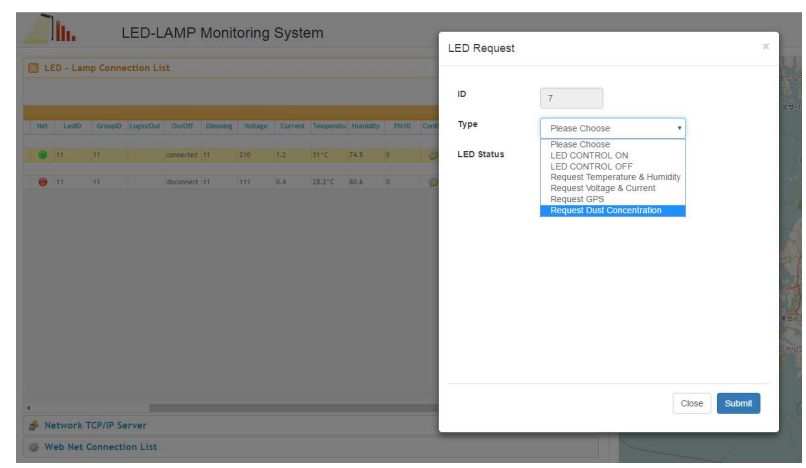

Fig. 12 Screenshot for type of request to the hardware page 


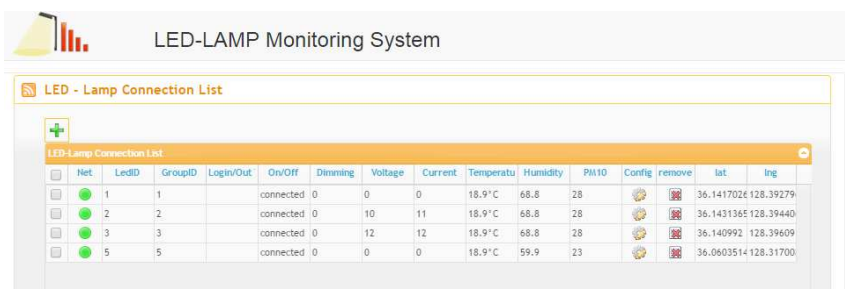

Fig. 13 Screenshot of the main page

Algorithm 1 shows the state of temperature and humidity of all points of the smart street light LED that is based on the predetermined coordinates. Here we use SKPlanet weather information as a microservice. We set every minute provision of information updates from SKPlanet to a webbased system.

\begin{tabular}{ll}
\hline & Algorithm 1. Showing weather condition \\
\hline 1 & DEFINE LEDlamp \\
2 & SET latitude,longitude, groupid,ledid \\
3 & BEGIN \\
& \\
4 & Function getWeatherCondition \\
5 & Pass In: integer representing longitude and latitude \\
6 & Using Korean Open API to get temperature and humidity \\
7 & API URL: http://apis.skplanetx.com/weather/current/minutely? \\
8 & Pass Out: temperature, humidity \\
9 & Endfunction \\
10 & Begin Algorithm 2 \\
11 & Begin Algorithm 3 \\
12 & END \\
\hline
\end{tabular}

Algorithm 2 shows the state of the particulate matter (PM) 10 dust concentration from all points of smart street light LED that is based on the predetermined coordinates. Here we use AirKorea weather information as a microservice.

\begin{tabular}{cc}
\hline & Algorithm 2. Showing dust concentration \\
\hline 1 & Function getDustConcentration \\
2 & Pass In: integer representing longitude and latitude \\
3 & Using Korean Open API to get PM10 Dust Concentration \\
4 & API URL: \\
& http://openapi.airkorea.or.kr/openapi/services/rest/ArpltnInfo \\
& rInqireSvc/getMsrstnAcctoRltmMesureDnsty? \\
5 & Pass Out: pm10 (dustconcentration) \\
6 & Endfunction \\
\hline
\end{tabular}

Algorithm 3 shows the state of the weather information from all points of smart street light LED that is based on the predetermined coordinates. We use SKPlanet weather information as a microservice. We use Weather News Flash (alert51) to show the condition of the weather was sunny, rainy, foggy, cloudy, storm or appropriate information on SKPlanet weather information.

\begin{tabular}{ll}
\hline \multicolumn{3}{c}{ Algorithm 3. Showing weather information } \\
\hline 1 & Function getWeatherNewsFlash \\
2 & Pass In: integer representing longitude and latitude \\
3 & Using Korean Open API to get Weather News Flash \\
& Information \\
4 & API URL: http://apis.skplanetx.com/weather/severe/alert? \\
6 & Pass Out: Weather News Flash (alert51) \\
\hline
\end{tabular}

\section{Analysis}

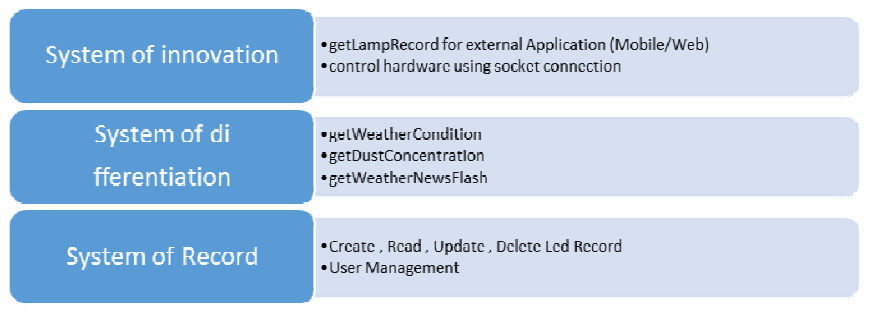

Fig. 14 Analyses of the system

In our implementation, we built micro services that consist of three layers of applications, namely the recording system, the differentiation system, and the system of innovation (as shown in Fig. 14). In the system of recording is the input made by the administrator: Create, Read, Update, Delete Led Record, and User Management. In the system of differentiation, we combine the real-time system with microservices which will always update the weather condition, weather information, and dust concentration. In the system of innovation, we combine the two directions; the first is communication towards the mobile / web service and the second is the control hardware by using socket communication.

From the three layer system that we built, all the elements can be run perfectly, the system can receive updates in real time, and administrators to communicate with the hardware in real-time as well.

\section{CONCLUSION}

In this paper, we implemented a project work on weather data aware remote monitoring for correlated color temperature LED streetlight system on the realization of IoT. In particular, we used ZigBee based wireless sensor nodes on each streetlight to provide periodic updates of temperature, humidity, and weather data to the server through TCP/IP communication protocol. We also utilized public weather to back up our sensor input data.

We follow agile methods to develop our software application. We chose this method because it delivers data faster than the traditional waterfall method. In addition, in the case of sudden changes, it can be continuously improved without changing the master data. Moreover, we successfully built software that is capable of utilizing three microservices and connect with several wireless sensor nodes. We further demonstrated a step-by-step algorithm development of weather information along with results.

As a future research work, we will work to enhance our system in order to support multiple platforms and to deliver multiple services.

\section{ACKNOWLEDGMENT}

This work was supported by the Kumoh National Institute of Technology.

\section{REFERENCES}

[1] Lopez Research, "An Introduction to the Internet of Things (IoT)", November 2013 
[2] Gubbi, Jayavardhana, et al. "Internet of Things (IoT): A vision, architectural elements, and future directions." Future Generation Computer Systems 29.7 (2013): 1645-1660.

[3] McEwen, Adrian, and Hakim Cassimally. Designing the internet of things. John Wiley \& Sons, 2013.

[4] Huaizhou Jin, "Research on the Lighting Performance of LED Street Lights With Different Color Temperatures".

[5] Kai Sorensen, Road lighting, NMF, 8 april 2013.

[6] BBC news. Two dead in 100-car South Korea pile-up. http://www.bbc.com/news/world-asia-31403615. Accessed: 2016-0701.

[7] Wolff, Eberhard. Microservices: Flexible Software Architecture Addison-Wesley Professional, 2016.

[8] Richardson, C. "Introduction to Microservices." (2015).

[9] WU Yue," Design of new intelligent street light control system"

[10] Y.M. Jagadeesh, Intelligent Street Lights

[11] Gul Shahzad, "Energy-Efficient Intelligent Street Lighting System Using Traffic-Adaptive Control"

[12] A. Lavric, V. Popa and I. Finis, "The Design of a Street Lighting Monitoring and Control System," in 2012 International Conference and Exposition on Electrical and Power Engineering, Iasi, 2012.

[13] C. Jing, D. Shu and D. Gu, "Design of Streetlight Monitoring and Control System Based on Wireless Sensor Networks," in 2007 2nd IEEE Conference on Industrial Electronics and Applications, Harbin, 2007.

[14] Mendalka, Maciej, et al. "WSN for intelligent street lighting system." Information technology (ICIT), 2010 2nd international conference on. IEEE, 2010.

[15] Leccese, Fabio, and Zbigniew Leonowicz. "Intelligent wireless street lighting system." Environment and Electrical Engineering (EEEIC), 2012 11th International Conference on. IEEE, 2012.

[16] Lian, Li, and Li Li. "Wireless dimming system for LED Street lamp based on ZigBee and GPRS." System Science, Engineering Design and Manufacturing Informatization (ICSEM), 2012 3rd International Conference on. Vol. 2. IEEE, 2012.

[17] N. Yoshiura, Y. Fujii and N. Ohta, "Smart Street Light System Looking Like Usual Street Lights Based on Sensor Networks," in 2013 13th International Symposium on Communications and Information Technologies, Surat Thani, 2013.

[18] Y. Fujii, N. Yoshiura, A. Takita and O. Naoya, "Smart Street Light System with Energy Saving Function Based on the Sensor Network," in Fourth International Conference on Future Energy Systems, Berkeley, 2013.

[19] F. Leccese and Z. Leonowicz, "Intelligent Wireless Street Lighting System," in 2012 11th International Conference on Environment and Electrical Engineering, Venice, 2012.

[20] F. Leccese, "Remote-Control System of High Efficiency and Intelligent Street Lighting Using a ZigBee Network of Devicesand Sensors," IEEE Transactions on Power Delivery, vol. 28, no. 1, pp. 21-28, 2013.

[21] C.-1. Fan and Y. Guo, "The Application of a ZigBee Based Wireless Sensor Network in the LED Street Lamp Control System," in 2011 International Conference on Image Analysis and Signal Processing, Wuhan, 2011.

[22] Z. Kaleem, I. Ahmad and C. Lee, "Smart and Energy Efficient LED Street Light Control System Using ZigBee Network," in 2014 12th International Conference on Frontiers of Information Technology, Islamabad, 2014.

[23] P. T. Daely, S. P. Heo and S. Y. Shin, "WSN Based LED Street Light System Prototype," in KICS Winter General Conference 2016, Jeongsun, 2016.

[24] P. T. Daely, G. B. Satrya and S. Y. Shin, "Implementation and Analysis of Real Time Scheduling for IoT: A Case Study of Smart LED Street Light," in KICS Summer General Conference 2016, Seogwipo, 2016.

[25] Atmel Corporation, "Atmel ATmega640/V- 1280/V-1281/V-2560/V2561/V Datasheet," Atmel Corporation, San Jose, 2014.

[26] Richtek Technology Corporation, "3A, 36V, 500kHz Synchronous Step-Down Converter," Richtek Technology Corporation, Chupei, 2015 .

[27] Sureshchandra, Kalpana, and Jagadish Shrinivasavadhani. "Moving from waterfall to agile." Agile, 2008. AGILE'08. Conference. IEEE, 2008 .

[28] Abrahamsson, Pekka, et al. "Agile software development methods: Review and analysis." (2002).

[29] Mashal Alqudah and Rozilawati Razali, "A Review of Scaling Agile Methods in Large Software Development," International Journal on Advanced Science, Engineering and Information Technology, vol. 6 , no. 6, 2016

[30] Shilpa Bahlerao and Maya Ingle, "Generalized Agile Estimation Method," International Journal on Advanced Science, Engineering and Information Technology, vol. 1, no. 3, pp. 262-267, 2011 